\title{
Pyruvate Kinase Deficiency along with Detection of Plasminogen Activator Inhibitor (PAI-1) 4G/4G Homozygous Genotype in Pregnancy
}

\author{
Stamatia Theodoridou ${ }^{1}$, Leonidas Zepiridis ${ }^{2}$, Alexandra Agapidou ${ }^{3}$, Theodoros \\ Theodoridis ${ }^{4}$ and Timoleon - Achilleas Vyzantiadis ${ }^{5}$ \\ ${ }^{1,3}$ Hemoglobinopathy Prevention Unit, Thessaloniki, Greece \\ ${ }^{2,4}$ First Obstetric and Gynecological Department, Aristotle University of Thessaloniki, Greece \\ ${ }^{5}$ First Department of Microbiology, Medical School, Aristotle University of Thessaloniki, Greece
}

Correspondence should be addressed to: Stamatia Theodoridou; hmesogiaki@ippokratio.gr

Received date: 7 May 2014; Accepted date: 7 October 2014; Published date: 28 December 2015

Academic Editor: Sophia Kitsiou-Tzeli

Copyright (C) 2015. Stamatia Theodoridou, Leonidas Zepiridis, Alexandra Agapidou, Theodoros Theodoridis and Timoleon - Achilleas Vyzantiadis. Distributed under Creative Commons CC-BY 4.0

\begin{abstract}
The most frequent enzyme abnormality of the glycolytic pathway which causes hereditary non-spherocytic hemolytic anemia is red cell pyruvate kinase (PK) deficiency.The disease is transmitted as an autosomal recessive trait. The case of a successful pregnancy in a 38 yearold woman with Pyruvate Kinase deficiency along with detection of plasminogen activator inhibitor (PAI-1) 4G/4G homozygous genotype is reported. The pregnancy was uneventful and a healthy girl was born.
\end{abstract}

Key words: Genetic Counseling, Hematologic and Clotting Problems, Recurrent pregnancy loss

\section{Introduction}

The most frequent enzyme abnormality of the glycolytic pathway which causes

Valentine et al (2) first reported this deficiency. Pyruvate kinase catalyzes the conversion of phosphoenolpyruvate and ADP to pyruvate and adenosine trisphosphate (ATP) in the EmbdenMeyerhoff glycolytic pathway and provides hereditary non-spherocytic hemolytic anemia is red cell pyruvate kinase (PK) deficiency. Hemolysis varies and can be very serious or milder (1).

the major source of energy in the erythrocyte. The enzyme deficiency leads to the accumulation of 2, 3diphosphoglycerate (DPG) and phosphoenolpyruvate which are the glycolytic pathway intermediates that

Cite this Article as: Stamatia Theodoridou, Leonidas Zepiridis, Alexandra Agapidou, Theodoros Theodoridis and Timoleon - Achilleas Vyzantiadis (2015), " Pyruvate Kinase Deficiency along with Detection of Plasminogen Activator Inhibitor (PAI-1) 4G/4G Homozygous Genotype in Pregnancy " Obstetrics \& Gynecology: An International Journal, Vol. 2015(2015), Article ID 744272,

DOI: $10.5171 / 2015.744272$ 
predispose to increased susceptibility to red cell hemolysis (3). The disease is transmitted as an autosomal recessive trait, clinical symptoms usually occur in compound heterozygotes for two mutant alleles and in homozygotes. The homozygote carriers require regular transfusions from early childhood while the heterozygotes experience clinical manifestations only under special conditions like infection or pregnancy. PK deficiency has a world-wide distribution. The prevalence of PK deficiency, as assessed by gene frequency studies, has been estimated to be $1: 20.000$ in the general white population. Recurrent pregnancy losses (RPL) have been connected with gene variants connected to thrombophilia and hypercoagulable state. There is a possible role of the PAI-1 $4 \mathrm{G} \backslash 5 \mathrm{G}$ gene variant that may be associated to hypofibrinolysis. There are some reports that underline the association between $4 G \backslash 4 G$ genotype of PAI-1 and recurrent pregnancy loss (RPL) $(4,5)$ and this association seems to be relevant if thromboembolism (VTE) is also present (6). The need for more data based on larger population screening is important.

We report the case of a young woman in her second pregnancy suffering from congenital pyruvate kinase deficiency and being homozygous carrier of the polymorphism of PAI- 1 . She underwent a thrombophilia examination since she already had one miscarriage.

\section{Case Report}

A 39-year-old woman in her second pregnancy (9th week of gestation) was referred to the Hemoglobinopathy Prevention Unit for genetic counseling as she was diagnosed with pyruvate kinase deficiency from the age of 13. She previously had undergone cholecystectomy, but not splenectomy. The proposita's hematological parameters were as follows: $\mathrm{Hb}: 9.3 \mathrm{~g} / \mathrm{dL}, \mathrm{RBC}: 2.45 \mathrm{x} 10$ 6/ $\mu \mathrm{L}, \mathrm{MCV}: 114 \mathrm{fL}, \mathrm{MCH}: 38$ pg, RDW: $15.4 \%$, reticulocytes:15\%, ferritin:730 $\mathrm{ngr} / \mathrm{ml}$ (normal range=20-81). Microscopic examination of peripheral blood smear showed anisocytosis and tear cells. Biochemical analysis of hemoglobin using high-performance liquid chromatography (HPLC) showed normal values for hemoglobin A2 and hemoglobin F. A maternal echo cardiogram was normal, ejection fraction (EF):75\%. We assessed hemoglobin levels every 3 weeks. At 28 weeks of gestation, hemoglobin was 8.3 gr/dl and suffered of palpitations, dyspnea and easy fatigability, so she was transfused with one unit of red cell. The patient also received folic acid $1 \mathrm{mg}$ but did not need iron supplementation due to increased ferritin levels (730ng/ml) due to chronic hemolysis. Fetal growth and well-being were assessed by monthly growth ultra sonography beginning at 20 weeks and weekly biophysical profiles at 32 weeks. The patient was on therapeutic dose of low molecular weight heparin (LMWH), as she was homozygous for $4 \mathrm{G} / 4 \mathrm{G}$ allele. This results in $25 \%$ higher levels of PAI-1 without being clarified from the literature whether it can lead to thrombosis, miscarriage or pre-eclampsia. After all, pregnancy itself increases the levels of PAI1. However, due to her first pregnancy loss at 12 weeks of gestation, age and due to her chronic hemolysis, she was put on low molecular heparin (prophylactic dose). During 37 weeks of gestation, the patient had a programmed caesarean delivery. Mother and infant had uncomplicated hospital courses.

\section{Discussion}

Pyruvate kinase deficiency is inherited in an autosomal recessive manner. More than 130 different mutations at the chromosome 1q21 locus are responsible for the variable clinical severity of hemolysis. Age at onset of symptoms and severity of anemia are related to the specific mutations mechanism of action and homozygosity compared with compound heterozygosity (7).

The enzymatic deficiency leads to a red cell susceptibility to hypoxic and conformational stress in the spleen and 
reticuloendothelial system. Definitive diagnosis is made by demonstrating pyruvate kinase deficiency using standardized assays. Patients with Pyruvate Kinase (PK) deficiency are transfusion dependent to varying degrees. An individual's anemia severity tends to remain constant except in cases related to infection or pregnancy. Splenectomy for individuals requiring frequent transfusion results in a $1-3 \mathrm{~g} / \mathrm{dL}$ rise in hemoglobin, alleviating hemolysis, and usually avoiding further transfusion. Cholecystectomy is performed for pigment stones or coincidental to splenectomy (7).

Depending on the history of splenectomy, transfusion, and iron overload, echocardiography is indicated as it assesses myocardial hypertrophy secondary to increased cardiac output compensating for chronic anemia (8). In our case, the estimated EF was normal. Folic acid, but not iron supplementation, is indicated. Splenectomy is indicated in very young patients requiring frequent transfusions, in patients who do not tolerate anemia, or in conjunction with cholecystectomy. The patient described did not have a splenectomy as anemia was well tolerated. Indications for red cell transfusion in pregnancy are controversial and unsupported by evidence. Some authors suggest that transfusion should be administered only for symptomatic anemia or in preparation for surgery or pregnancy (9). Patients with pyruvate kinase deficiency have increased red cell 2,3 diphosphoglycerate, decreased hemoglobin oxygen affinity, and increased oxygen release to tissues. Thus, patients exhibit greater tolerance to increasing oxygen demand than expected on the basis of the hemoglobin. Other authors recommend transfusions to maintain a hemoglobin concentration of $7-8 \mathrm{mg} / \mathrm{d}$ L or more $(10$, 11, 12).

Transfusion therapy does not seem to mitigate neonatal outcome. Specifically, while all three reported pregnancies without transfusion $(9,12)$ resulted in growth-restricted fetuses, so did two of three pregnancies managed with transfusions (11). Pregnant women with pyruvate kinase deficiency should be monitored for hypertensive disorders, observed in three of seven reported cases $(10,12)$. Finally, it is recommended monthly to perform ultrasonographic assessments of fetal growth after 20 weeks, because 5 of 6 affected pregnancies experienced intrauterine growth restriction.

Recurrent pregnancy loss (RPL) represents a major health problem in up to $5 \%$ of women of reproductive age (13). Inherited thrombophilia agents are reported as one of the main causes of RPL especially if several diseases responsible of potential RPL are excluded (an-ovulation, ovarian dysfunction, hypopituitarism and diabetes, uterine malformation, genetic alterations, inflammatory diseases) $(14,15,16,17,18)$.

Established inherited thrombophilic risk factors include the G1691A mutation of the Factor V (Leiden), the G20210A mutation of the prothrombin gene, and hyperhomocysteinemia. The association between homozygosity for the C677T polymorphism of the methylene-tetrahydrofolate reductase (MTHFR) gene and the $4 \mathrm{G} / 5 \mathrm{G}$ polymorphism of the plasminogen activator inhibitor (PAI-1) gene promoter with thrombosis is controversial. So far, there is no information whether severe pre-eclampsia is related to these risk factors. Preeclampsia and certain variants of the genes coding for angiotensin-converting enzyme, CTLA-4, thrombin, factor $\mathrm{V}$, lipoprotein lipase and plasminogen activator inhibitor1 are related. As for the rare thrombophilic defects like dysfibrinogenemia, elevated TAFI, elevated PAI-1, t-PA deficiency, only few reported cases exist. The G20210A prothrombin gene mutation and the PAI-1 5G/5G genotype seem to be risk factors inducing pre-eclampsia in women with already existing fetomaternal dysfunction. Among women with G20210A and PAI-1 $5 \mathrm{G} / 5 \mathrm{G}$, genotype earlier onset of preeclampsia among women is reported (18).

In our case, the patient was diagnosed with PAI-1 polymorphism and 4G/4G 
homozygous genotype. Taking under consideration age and past medical record, she was treated with LMWH. She was monitored for pre-eclampsia which was not detected.

According to the type of thrombophilia, the patient's additional risk factors and co morbidities, the treatment should be individualized. Women with pyruvate kinase deficiency should be counseled before the pregnancy. They should be informed about the risks of hemolysis and the potential need of blood transfusion. There are few reported cases of pregnancy in women with pyruvate kinase deficiency in the literature. This is the first case reporting pregnancy in a woman with pyruvate kinase deficiency and PAI-1 polymorphism which had a positive outcome both for mother and child.

\section{Disclosure}

We declare that all the authors do not have any relationships with companies that may have financial information contained in the manuscript.

\section{References}

1. Zanella A, Elisa Fermo E, Paola Bianchi P, Valentin G. Red cell pyruvate kinase deficiency: molecular and clinical aspects. Brit J. Haematology, 2005;130: 11-25.

2. Valentine $\mathrm{WN}$, Tanaka KR, Miwa S. A specific erythrocyte glycolytic enzyme defect in three subjects with congenital non spherocytic hemolytic anemia. Trans Assoc Am Phys, 1961;74:100-10.

3. Wax J, Pinette M, Cartin A, Blackstone J. Pyruvate kinase deficiency complicating in pregnancy. Obst \& Gyn. 2007;2:109.

4. Goodman CS, Coulam CB, Jeyendran RS, Acosta VA, Roussev R. Which thrombophilic gene mutations are risk factors for recurrent pregnancy loss? Am J Reprod Immunol 2006;56(4):230-236.

5. Coulam CB, Jeyendran RS, Fishel LA, Roussev R. Multiple thrombophilic gene mutations rather than specific gene mutations are risk factors for recurrent miscarriage. Am J Reprod Immunol 2006;55(5):360-368.

6. Coulam CB, Wallis D, Weinstein J, DasGupta DS, Jeyendran RS. Comparison of thrombophilic gene mutations among patients experiencing recurrent miscarriage and deep vein thrombosis. Am J Reprod Immunol 2008;60(5):426-431.

7. Zanella A, Bianchi P. Red cell pyruvate kinase deficiency: from genetics to clinical manifestations..Baillieres Best Pract Res Clin Haematol 2000;13:57-81.

8. Dolan LM, Ryan M, Moohan J. Pyruvate kinase deficiency in pregnancy complicated by iron overload.. B J O G 2002;109:844-6.

9. Ghidini A, Korker VL. Severe pyruvate kinase deficiency anemia: a case report. J Reprod Med 1998;43:713-715.

10.Amankwah KS, Dick BW, Dodge S. Hemolytic anemia and pyruvate kinase deficiency in pregnancy. Obst Gyn1980;55:42S-45S.

11.Fanning J, Hinkle RS. Pyruvate kinase deficiency hemolytic anemia: two successful pregnancy outcomes. Am J Obst Gynl 1985;153:313-4.

12.Sarig G, Younis JS, Hoffman R, Lanir N, Blumenfeld Z, Brenner B Thrombophilia is common in women with idiopathic pregnancy loss and is associated with late pregnancy wastage. Fertil Steril 2002;77(2):342-347.

13.Eldor A. Thrombophilia, thrombosis and pregnancy. Thromb Haemost 2001;86(1):104-111.

14.Carp H, Salomon O, Seidman D, Dardik $\mathrm{R}$, Rosenberg N, Inbal A. Prevalence of genetic markers for thrombophilia in recurrent pregnancy loss. Hum Reprod 2002;17(6):1633-1637. 
15.Prandoni P, Tormene D, Simioni P, Girolami A. Venous thromboembolism, fetal loss and preeclampsia in pregnant women with congenital thrombophilia. Clin Lab 2001;47(3-4):155-159.

16.Di Micco P, D’Uva M, Strina I, De Placido G, Di Fiore R, Quaranta S, Castaldo G. Recurrent pregnancy loss and thrombophilia. Clin Lab 2007;53(5-6):309314.
17.Abbate R, Lenti M, Fatini C, Gazzini A, Lapini I, Fedi S. L'ipercoagulabilità gravidica e puerperale. Haematologica 2003;88 (Suppl.7):1-2

18.Gerhardt A, Goecke TW, Beckmann MW, Wagner KJ, Tutschek B, Willers R. The G20210A prothrombin-gene mutation and the plasminogen activator inhibitor (PAI-1) 5G/5G genotype are associated with early onset of severe pre-eclampsia. J Thromb Haemost. 2005 Apr;3(4):686-91. 
Stamatia Theodoridou, Leonidas Zepiridis, Alexandra Agapidou, Theodoros Theodoridis and Timoleon Achilleas Vyzantiadis (2015), Obstetrics \& Gynecology: An International Journal,

DOI: $10.5171 / 2015.744272$ 